\title{
POSTURAL STABILITY, PHYSICAL ACTIVITY, AEROBIC CAPACITY AND THEIR ASSOCIATIONS FOR YOUNG PEOPLE WITH AND WITHOUT INTELLECTUAL DISABILITIES
}

\author{
Sven Blomqvist ${ }^{*}$ \\ Swedish Development Centre for Disability Sport, Bollnäs, Sweden \\ Department of Community Medicine and Rehabilitation, Physiotherapy, Umeå University, Umeå, Sweden \\ Anita Wester \\ Department of Research and Evaluation, Swedish National Agency for Education, Stockholm, Sweden \\ Elin Persson, Hillevi Sundkvist, Gunnevi Sundelin and Börje Rehn \\ Department of Community Medicine and Rehabilitation, Physiotherapy, Umeå University, Umeå, Sweden
}

*Corresponding author: Swedish Development Centre for Disability Sport, Box 1002, SE-821 11 Bollnäs, Sweden. E-mail: sven.blomqvist@suh.se

Previous studies show that people with intellectual disability (ID) appear to have impaired postural stability, a lower level of physical activity, and lower aerobic capacity compared to persons without ID, limitations that could affect their health. This study investigates these physical functions and their associations in a group of young people with ID compared to an age-matched group without ID. In total, this cross-sectional study included 106 high school students (16-20 years): 57 students with mild to moderate ID and 49 agematched students without ID (control group). Tests were performed for postural stability, level of physical activity, and aerobic capacity. Both females and males with ID had significantly lower estimated maximum oxygen uptake $(1 \mathrm{O} 2 / \mathrm{min})(\mathrm{p}<0.001$ for females and $\mathrm{p}=0.004$ for males) and a lower aerobic capacity expressed relative to body weight $\left(\mathrm{ml} \mathrm{O} 2 / \mathrm{kg}^{*} \mathrm{~min}\right)(\mathrm{p}<0.001$ for females and $\mathrm{p}=0.012$ for males) compared to age-matched peers. Analyses of associations were made using the Pearson's correlation coefficient and multivariate linear regression analysis. No significant associations could be found. Physical status appears impaired for young people with ID and functions, such as postural stability, should be evaluated separately.

Keywords: Mental retardation, physical capacity, motor activity, postural equilibrium, youth, teenagers

\section{INTRODUCTION}

Postural stability, physical activity, and aerobic capacity will likely influence physical well being (World Health Organization, 2010). People with intellectual disability (ID) appear to have impaired postural stability, a lower level of physical activity, and a lower aerobic capacity compared to the general population, limitations that seem to exist throughout their life (Blomqvist, Wester, Sundelin, \& Rehn, 2012; Dellavia, Pallavera, Orlando, \& Sforza, 2009; Hale, Miller, Barach, Skinner, \& Gray, 2009; Hinckson \& Curtis, 2013; Lahtinen, Rintala, \& Malin, 2007; Salaun \& Berthouze-Aranda, 2012; Suomi \& Koceja, 1994; Wallen, et al., 2009). It could be hypothesised that postural stability should be associated with level of physical activity and aerobic capacity; however, the associations among these functions have not previously been investigated for young people with ID.

To be diagnosed with an ID, the intelligence level (IQ) has to be below average, $<70$. Furthermore, the person must have significant difficulties with adaptive behaviour needed for everyday living. In addition, the diagnosis should be determined before the age of 18 . The disability and presumptive lack of knowledge on how to live a healthy lifestyle makes it difficult for these individuals to live an ordinary life and could negatively affect their physical condition (Krahn, Hammond, \& Turner, 2006; Walsh, Kerr, \& van Schrojenstein Lantman-de Valk, 2003).

To participate in physical activity and sports, it is necessary to control postural stability (Ulrich \& Ulrich, 1985). Postural stability can be defined as the ability to maintain the vertical projection 
of the body's centre of mass (CoM) within the base of support (BoS). To stabilise the CoM, the central nervous system integrates sensory information received from visual, vestibular, and somatosensory inputs. The information can be used to determine the proper motor commands to be sent to the muscles (Shumway-Cook \& Woollacott, 2012). Movement of CoM can be indirectly measured by analysing the location of the resulting ground reaction force, the centre of pressure (CoP). Several studies show an impaired postural stability in groups of persons with ID compared to persons without ID (Blomqvist et al., 2012; Dellavia et al., 2009; Haleet al., 2009; Lahtinen et al., 2007; Suomi \& Koceja, 1994). One study of adults with ID showed that physical activity positively affects postural stability (Bartlo \& Klein, 2011); another study of adults with ID showed that physical activity can improve aerobic capacity and endurance (Hottenrott, Ludyga, \& Schulze, 2012).

A review of literature suggests that children and young people with ID are significantly less physically active than young people without ID (Hinckson \& Curtis, 2013). The World Health Organization (WHO) recommends that children and adolescents (5-17 years) should do at least 60 minutes of moderate intensity aerobic activity every day and persons between 18-64 years should do at least 150 minutes of moderate intensity physical activity per week or a total of 75 minutes of vigorous physical activity per week. These guidelines were determined to help people become or maintain good physical fitness so as to avoid diseases associated with a sedentary lifestyle such as diabetes mellitus, obesity, or coronary heart disease. Regular physical activity has shown to improve strength, physical capacity, overall health, and quality of life (World Health Organization, 2010).

Aerobic capacity, the ability of the circulatory and respiratory systems to supply enough oxygen to skeletal muscles during sustained physical activity (Wilmore \& Costill, 1999), seems to be one of the most important components of health (Fogelholm, 2010). Research about aerobic capacity for young people with ID seems to be contradictory. Some studies have shown that the aerobic capacity is significantly lower among young people with ID compared to young people without ID (Salaun \& Berthouze-Aranda, 2012; Wallen et al., 2009), but another study found that there was no difference between young people with and without ID(Baynard, Pitetti, Guerra, Unnithan, \& Fernhall, 2008).

The associations between postural stability, level of physical activity and aerobic capacity has to our knowledge not been investigated for people with ID, but a study of older people found a strong association between aerobic capacity, balance, movement speed and cognitive function (Voelcker-Rehage, Godde, \& Staudinger, 2010). Therefore, the present study investigates postural stability, level of physical activity, and aerobic capacity and their associations for young people with and without ID. We hypothesised that there is an association between better postural stability, high level of physical activity, and high aerobic capacity in both groups.

\section{METHODS}

\section{Recruitment}

A cross-sectional study design was implemented. The participants were recruited from a pool of 500 young people who volunteered for an earlier study (Blomqvist et al., 2012). The volunteers were from two upper secondary schools in Bollnäs, Sweden: a special school for young people with an intellectual disability (ID) and a regular school. From this pool of volunteers, 58 students from the special school and 50 students from the regular school were randomly chosen. In the ID group, more participants were recruited because through experience it was known that they tend to have more dropouts. All participants were informed about the study and the tests, both verbally and in writing, and then asked if they wanted to participate. Those with an ID who were under 18 years old needed their parents' approval to take part in the study. A letter that included information about the study and 'an agreement to participate form' to be signed was sent home to the parents. To have a power of $80 \%$ and a level of significance of 0.05 to distinguish between the two groups (ID, non-ID) with 
regards to postural stability, 40 individuals were needed in each group when variance (based on earlier measurements, (Blomqvist et al., 2012)) of sway velocity was used.

\section{Participants}

All participants agreed to participate and in total, 108 young people were included in the study, but before the test started, one person from each group dropped out, so the final total was 57 with ID (26 males and 31 females) and 49 without ID (27 males and 22 females). The inclusion criteria for the ID group were: a) an ID that was mild (IQ 50-69) to moderate (IQ 35-49), b) the ID should have been diagnosed before 18 years of age, and c) they were assessed as having significant limitations in adaptive behaviour: conceptual, social, and practical skills. All participants in the ID group were diagnosed by a registered psychologist, using an intellectual functioning test (IQ-test) and standardised tests that determine adaptive limitations. The exclusion criteria for both groups were neurological signs or recent injury in the lower extremities (the ID group was tested for loss of reflexes, sensibility, and isometric strength), need of walking aids, previous or on-going vestibular neuritis, and a diagnosis of cerebral palsy. Their sight was tested using an eye chart (a visual acuity of better than 0.1 was required for participation). The non-ID participants were significantly taller $(4-5 \mathrm{~cm})$ than the ID participants. No significant differences in body mass index (BMI) were found (Table 1).

\section{Procedure}

Their height and weight was assessed and their age was noted. Physical activity was measured with the help of a pedometer. Within two months after the pedometer testing, a test for aerobic capacity and five postural stability tests were conducted. Additionally, the ID participants were given the vision test and screened for loss of reflexes, sensibility, and isometric strength. Next, the postural stability tests were randomly conducted and these tests were followed by the aerobic capacity test.

\section{Postural stability tests}

Five different postural stability tests were executed on a force-platform (MuscleLab model ET-FPL-1) for 30 seconds. The sway velocity of the $\mathrm{CoP}$ was measured in $\mathrm{mm} / \mathrm{s}$ by MuscleLab (Model 4000e). Previous studies have shown that measure of CoP sway velocity has high reliability (Lafond, Corriveau, Hebert, \& Prince, 2004; Salavati et al., 2009). The participants were all barefoot during the tests, standing with their feet close together to minimize their BoS. Five postural stability tests were conducted: 1) standing with eyes open; 2) wearing blackened, blindfolding glasses; 3 ) eyes open on a soft Airex mat $2.5 \mathrm{~cm}$ (Airex AG); 4) blindfolded on a soft Airex mat $2.5 \mathrm{~cm}$ (Airex AG); and 5) eyes open, standing while rotating the head $30^{\circ}$ to the left and $30^{\circ}$ to the right at $60^{\circ} / \mathrm{second}$. To make sure the participants rotated their head $30^{\circ}$ to each side, two poles were placed at the correct angles in front of the test person. The participants were instructed to point their nose to each pole to the pace of a metronome. If someone had trouble keeping up with the pace of the metronome, the test leader helped by moving a hand in front of the test person as guidance. To avoid systematic bias, the order of the postural stability tests for each participant was randomised. The tests were made three times with a 30 -second break between each test. There was no trial run. The best result, the one with the least CoP sway velocity, of the three trials was recorded. Due to various reasons such as withdrawal and sickness, seven participants did not perform the postural stability tests.

\section{Physical activity}

To measure the level of physical activity, the participants wore a pedometer for five consecutive days (Sunday through Thursday in February) to measure the total numbers of steps taken per day. The pedometer types were Keep Walking LS2000 and LS7000 (Yamax SW200/ LS2000). Previous studies have shown that the Yamax SW series has a good validity (ICCIntraclass Correlation Coefficients $(95 \% \mathrm{CI})=0.98$ (0.94-0.98) and reliability (Chronbach's Alpha = 
0.992)(Crouter, Schneider, Karabulut, \& Bassett, 2003; Schneider, Crouter, Lukajic, \& Bassett, 2003). The participants were given a diary and instructed to write down how many steps they took each day, if they were sick on any day, or if the pedometer did not work on any day. All the participants received information on how to use the pedometer: wear it during all waking hours of the day; wear in the waistband just above the knee; wear it during all activities of the day (except if swimming or participating in other water activities); and every night record the steps taken in the diary, take the pedometer off, and reset it. The participants who only had two days or fewer registered were excluded as well as the ones who did not have any result registered from Sunday. Sunday was considered an important day because the activity level was in many cases different this day. Due to this, ten participants were excluded. Previous studies have shown that at least three days of measurements are necessary to obtain a valid result (Temple \& Stanish, 2009; Tudor-Locke et al., 2005). The number of total steps taken during the five-day test period was divided by how many days the test person had a registered result (number of total steps/day).

\section{Aerobic capacity}

To estimate aerobic capacity, the ÅstrandRhyming sub maximal cycle ergometer test was used. This test is well known and has been used in previous studies with good validity and reliability (Noonan \& Dean, 2000). Before the test, the participants were briefly interviewed to determine the level of their daily physical activity. Using this information, the tester determined the appropriate resistance. If they took any medication that could mislead the result of the test (beta-blockers, blood pressure adjusting medicine, etc.), their test results were not included in the analysis. Because the tests were made on a speed independent cycle (Monark 839E), the breaking effect remained consistent (constant power mode) irrespective of the speed of the pedals. This strategy made it easier to keep constant resistance even if the test person was not able to keep up with the pace. The test leader set the resistance at an adjusted level to fit the individual's physical capacity. The participant then cycled on a pace set by a metronome at 50 rpm with the given resistance. During the first two minutes, the test leader had the opportunity to alter the resistance for the test person to reach the right pulse frequency. For the test to be counted, the pulse had to reach at least 120 beats per minute. At the end of each minute, the pulse frequency was registered. To measure the pulse, a pulse band (Monark art.nr. 9303-95) was strapped over the test person's thorax. In some cases where the pulse band did not work due to a connection error, the test leader measured the pulse manually at the wrist (a.radialis), taking the pulse frequency for 30 seconds and then multiplying it by two to calculate beats per minute. An estimation of Borg's Rating of Perceived Exertion (RPE) scale was not included in this study because of the lack of understanding among the persons with ID. Based on the pulse and the resistance, maximum oxygen uptake (VO2max(l O2/min)) was predicted. The aerobic capacity expressed relative to body weight ( $\left.\mathrm{ml} \mathrm{O} 2 / \mathrm{kg}^{*} \mathrm{~min}\right)$ was also calculated by dividing the VO2max by the person's weight. Eight participants were excluded in the aerobic capacity test due to various reasons such as withdrawal, sickness, medication, or not reaching the predetermined pulse frequency (over 120 beats per minute).

\section{Data analysis}

To compare age and anthropometric characteristics, independent sample t-test was carried out for age, height, and BMI in the female group (with ID and without ID). The MannWhitney u-test was used for the comparison of weights because the data was not normally distributed. For comparison of the male groups, independent sample t-test was used for height, weight, and BMI, and the Mann-Whitney u-test was used when comparing age. The independent sample t-test was also used to compare results of postural stability, level of physical activity, and aerobic capacity between the two groups. Correlation analysis between postural stability, physical activity, and aerobic capacity was made using the Pearson's correlation coefficient (rp). 
A multiple linear regression analysis was used to evaluate the dependent variable postural stability influence from the independent variables: level of physical activity and aerobic capacity. $\mathrm{R}^{2}$ values were calculated for both groups. The following multiple linear regression model was made for both groups: Postural stability $=a+b_{1}{ }^{*}$ physical activity $+\mathrm{b}_{2} *$ aerobic capacity

Feet close together and standing with eyes open was used to represent postural stability in the multiple linear regressions analysis. Level of physical activity was divided into five groups (steps/day) according to Tudor-Locke et al.: 1. Very low $=0-5000 ; 2$. Low $=5001-7500 ; 3$. Average $=$ 7501-10,000; 4. High = 10 001-12,500; and 5. Very high > 12,501 (Tudor-Locke, Hatano, Pangrazi, \& Kang, 2008). Aerobic capacity was divided into five groups ( $\mathrm{ml} \mathrm{O} 2 / \mathrm{kg}^{*} \mathrm{~min}$ ) according to Åstrand (2012): Åstrand and colleagues tested the aerobic capacity in a group of people in Sweden (age between 20-29 yrs), then they divided the max VO2 with the body mass in $\mathrm{kg}$. From those test results they could divide the aerobic capacity expressed relative to body weight $\left(\mathrm{ml} \mathrm{O} 2 / \mathrm{kg}^{*} \mathrm{~min}\right)$ into five different groups. 1 . Very low $=0-28.0$ for women and 0-38.0 for men; 2 . Low $=28.1-34.0$ for women and 30.1-43.0 men; 3. Average $=34.1$ 43.1 for women and 43.1-51.0 for men; 4 . High = 43.2-48.0 for women and 51.1-56.0 for men; and 5 . Very high $=48.1$ and more for women and 56.1 and more for men (Åstrand, 2012). Significance level was set at $p$-value $<0.05$. To avoid mass significance for analyses of associations, the alphavalue was divided by the number of comparisons (alpha/n) according to Bonferroni $(0.05 / 15=$ $0.003)$. All statistical analyses were made with SPSS version 20 (SPSS Inc.).

\section{Ethics}

Approval of the study was obtained from the Regional Ethical Review Board in Umeå, Sweden (No. 09-076M).

\section{RESULTS}

Overall, the participants with ID had significantly higher sway velocity than peers without ID.
This pattern was significant for males in all tests ( $p$-values $<0.001-0.006$ ), but for females only feet together standing on a soft mat was sigificantly different (p-value 0.034). As a group, participants with ID were significantly less physically active. A gender stratification showed that young females with ID were less physically active than young females without ID ( $p$-value 0.001). However, no sigificant differences were seen between males with and without ID $(p$-value $=0.610)$. Young people with ID had significantly lower estimated maximum oxygen uptake compared to peers without ID ( $p$-value $<0.001$ for females; $p$-value 0.004 for males) and the ID group also had significantly lower scores for aerobic capacity expressed relative to body weight ( $p<0.001$ for females; $p=0.012$ for males) (Table 2).

No significant associations could be found between postural stability and level of physical activity and postural stability and aerobic capacity in the two groups (Table 3 ).

The results of the multiple linear regression analyses demonstrated that the model with all independent variables (physical activity and aerobic capacity) influence on postural stability was explained by $4.9 \%$ (p-value 0.298 ) for young people with ID $(\mathrm{n}=51)$ and by $7.3 \%$ (p-value $0.236)$ for young people without ID $(n=41)$. For women the influence was 4,9\% (p-value 0.696) for ID $(n=28)$ and $5.5 \%$ (p-value 0.586$)$ for nonID $(n=18)$, and for men the influence was $9.7 \%$ (p-value 0,138$)$ for ID $(n=23)$ and $3.1 \%$ (p-value $0.281)$ for non-ID $(n=23)$.

\section{DISCUSSION}

In this study, participants with ID had more postural sway, were less physically active and had lower aerobic capacity than age matched peers without ID. Females with ID had a lower level of physical activity and aerobic capacity than females without ID. Males with ID had more postural sway and lower aerobic capacity than males without ID. Physical activity and aerobic capacity had no influence on postural stability.

The results in this study are in line with other studies that have investigated postural stability 
by measuring postural sway (Blomqvist et al., 2012; Dellavia et al., 2009; Suomi \& Koceja, 1994; van Emmerik, Sprague, \& Newell, 1993). Hinckson and Curtis (2012) found that young people with ID were significantly less active than their peers without ID. The same pattern was shown in our study, but only for females. We have no explanation for the sex differences. Further studies with that focus appear necessary.

This study found that aerobic capacity among young people with ID was significantly lower than for peers without ID, a finding that is consistent with other studies that used sub maximal tests to test adolescents with ID (Salaun \& Berthouze-Aranda, 2012; Wallen et al., 2009). However, these results are not in line with Baynard et al. (2008); they reported a lower level for young people with ID, but it was not significant. This distinction could depend on group differences (e.g. age and sex). However, they did not report the group structure. Another reason could be that they used a maximal test on treadmill and the other studies used sub maximal tests. Our experience is that it is hard for young people with ID to do a maximal test fully because they have problems pushing themselves to exhaustion.

No significant associations between postural stability tests, level of physical activity, and aerobic capacity were seen. To our knowledge, the associations among these three physical functions have never been studied before in a group of young people with ID. A study of adults with ID by Bartlo \& Klein (2011) showed that physical activity is positively associated with postural stability, but this association could not be found in our study and the reason could be the age difference between groups. Some studies, however, have investigated this association on a general population of older physically active persons. Voelcker-Rehage et al. (2010) found a strong association between postural stability, aerobic capacity and cognitive functioning and a study by Hayashia, et al. (2012) on elderly (60> years old) did not detect any associations, but found that those with higher aerobic capacity had a better postural stability and a higher P-ADL (Personal activity in daily life). Nevertheless, other studies have have not found any associations (Hughes, Duncan, Rose, Chandler, \& Studenski, 1996; Spagnuolo, Jurgensen, Iwama, \& Dourado, 2010). Thus, results from studies on elderly seem contradictory and may not even be suitable for comparisons as the higher age could affect several organs and systems of the body resulting in lower strength, reduced nerve conduction velocity, and impaired vision (The Swedish National Institute of Public Health, 2010).

Despite that there were differences in aerobic capacity, physical activity and postural stability between the two groups no statistically justified explanation for the postural stability could be detected by the multiple linear regression models. To our knowledge, no other studies have investigated this for the same target group. However, Voelecker-Rehage et al. (2010) found a strong association between aerobic capacity, postural stability, movement speed and cognitive function for older people. The reason we did not find any associations could depend on that our subjects are young and the differences in early years are smaller.

\section{Methodological considerations}

The tests for postural stability were all carried out on a force-plate that measured the velocity of CoP sway, which has shown high validity (Lafond et al., 2004; Salavati et al., 2009). The different stability tests used are to various degrees challenging the various sensory subsystem, vision, vestibular, and somatosensory systems. One reason for not finding any associations might be that postural stability was measured during static conditions. There might be a limited carry-over effect from dynamic postural stability tasks performed during physical activity to a static balance test.

Pedometer testing is considered a good way to measure physical activity (Crouter, et al., 2003; Schneider, et al., 2003) and it is cheap, simple to use and understand. However, its accuracy is limited and pedometer testing does not register water activities or upper limb activities. Also activities such as cross-country skiing or horseback riding could alter the results. During 
the test period for physical activity, no such activities were done during school but could have occurred during leisure time. Another aspect to consider is that the young people were motivated by wearing the pedometer, which could have made them more active during the measurement period. Thus, an attention bias could have been introduced. Nevertheless, it should have affected both groups similarly.

The power of this study was based on differences between groups with regards to postural stability (sway). Forty subjects in each group were necessary. Stratification into sexes was made during the analysis which revealed important differences. Because of that, a type II error might occur. The interpretation of these results should therefore be interpreted cautiously.

\section{Perspectives}

It seems as if physical status can be improved for young persons with ID as they do not reach the level of their peers. Because of the lack of associations between postural stability, physical activity level, and aerobic capacity, these physical functions should be evaluated and exercised separately. Persons with ID might not be able to realise the health advantages of being physically active and therefore, it is important that they are provided support from parents, guardians, school staff, and others to encourage them to be more physically active, especially the females. This could promote their overall health and help them avoid diseases associated with a sedentary lifestyle.

\section{Conclusions}

Postural stability for males with ID and level of physical activity for females with ID were significantly lower compared to peers without ID. Aerobic capacity was significantly lower in both young men and women with ID compared to peers without ID. The present study did not find any associations between postural stability, level of physical activity, and aerobic capacity. It appears that postural stability is not associated with the physical status for young people with ID.

\section{REFERENCES}

Bartlo, P., \& Klein, P. J. (2011). Physical activity benefits and needs in adults with intellectual disabilities: systematic review of the literature. American Journal on Intellectual and Developmental Disabilities, 116, 220-232.

Baynard, T., Pitetti, K. H., Guerra, M., Unnithan, V. B., \& Fernhall, B. (2008). Age-related changes in aerobic capacity in individuals with mental retardation: a 20-yr review. Medicine and Science in Sports and Exercise, 40, 1984-1989.

Blomqvist, S., Wester, A., Sundelin, G., \& Rehn, B. (2012). Test-retest reliability, smallest real difference and concurrent validity of six different balance tests on young people with mild to moderate intellectual disability. Physiotherapy, 98, 313-319.

Crouter, S. E., Schneider, P. L., Karabulut, M., \& Bassett, D. R., Jr. (2003). Validity of 10 electronic pedometers for measuring steps, distance, and energy cost. Medicine and Science in Sports and Exercise, 35, 1455-1460.

Dellavia, C., Pallavera, A., Orlando, F., \& Sforza, C. (2009). Postural stability of athletes in Special Olympics. Perceptual Motor Skills, 108, 608-622.

Fogelholm, M. (2010). Physical activity, fitness and fatness: relations to mortality, morbidity and disease risk factors. A systematic review. Obesity Reviews, 11, 202-221.

Hale, L., Miller, R., Barach, A., Skinner, M., \& Gray, A. (2009). Motor Control Test responses to balance perturbations in adults with an intellectual disability. Journal of Intellectual and Development Disabilty, 34, 81-86.

Hinckson, E. A., \& Curtis, A. (2013). Measuring physical activity in children and youth living with intellectual disabilities: a systematic review. Research in Developmental Disabilities, 34, 72-86.

Hottenrott, K., Ludyga, S., \& Schulze, S. (2012). Effects og high intensity training and continous andurance training on aerobic and body composition in recreationally active runners. Journal of Sports Science and Medicine, 11, 483-488. 
Hughes, M. A., Duncan, P. W., Rose, D. K., Chandler, J. M., \& Studenski, S.A. (1996). The relationship of postural sway to sensorimotor function, functional performance, and disability in the elderly. Archives of Physical Medicine and Rehabilitation, 77, 567-572.

Krahn, G. L., Hammond, L., \& Turner, A. (2006). A cascade of disparities: health and health care access for people with intellectual disabilities. Mental Retardation and Developmental Disabilities Research Reviews, 12, 70-82.

Lafond, D., Corriveau, H., Hebert, R., \& Prince, F. (2004). Intrasession reliability of center of pressure measures of postural steadiness in healthy elderly people. Archives of Physical Medicine Rehabilitation, 85, 896-901.

Lahtinen, U., Rintala, P., \& Malin, A. (2007). Physical performance of individuals with intellectual disability: a 30 year follow up. Adapted Physical Activity Quarterly, 24, 125-143.

Noonan, V., \& Dean, E. (2000). Submaximal exercise testing: clinical application and interpretation. Physical Therapy, 80, 782-807.

Salaun, L., \& Berthouze-Aranda, S. E. (2012). Physical fitness and fatness in adolescents with intellectual disabilities. Journal of Applied Research in Intellectual Disabilities, 25, 231-239.

Salavati, M., Hadian, M. R., Mazaheri, M., Negahban, H., Ebrahimi, I., Talebian, S., et al. (2009). Test-retest reliability [corrected] of center of pressure measures of postural stability during quiet standing in a group with musculoskeletal disorders consisting of low back pain, anterior cruciate ligament injury and functional ankle instability. Gait and Posture, 29, 460-464.

Schneider, P. L., Crouter, S. E., Lukajic, O., \& Bassett, D. R., Jr. (2003). Accuracy and reliability of 10 pedometers for measuring steps over a 400-m walk. Medicine and Science in Sports and Exercise, 35, 1779-1784.

Shumway-Cook, A, \& Woollacott, M.H. (2012). Motor Control: Theory and Practical Applications. Baltimore, MD: Williams \& Wilkins.
Spagnuolo, D. L., Jurgensen, S. P., Iwama, A. M., \& Dourado, V. Z. (2010). Walking for the assessment of balance in healthy subjects older than 40 years. Gerontology, 56, 467-473.

Suomi, R., \& Koceja, D. M. (1994). Postural sway patterns of normal men and women and men with mental retardation during a two-legged stance test. Archives of Physical Medicine Rehabilitationl, 75, 205-209.

Temple, V. A., \& Stanish, H. I. (2009). Pedometer-measured physical activity of adults with intellectual disability: predicting weekly step counts. American Journal on Intellectual and Developmental Disabilities, 114, 15-22.

The Swedish National Institute of Public Health. (2010). Physical Activity in the Prevention and Treatment of Disease. Professional Associations for Physical Activity, Elanders; 2010. ISBN: 978-91-7257-715-2, 200-208.

Tudor-Locke, C., Burkett, L., Reis, J. P., Ainsworth, B. E., Macera, C. A., \& Wilson, D. K. (2005). How many days of pedometer monitoring predict weekly physical activity in adults? Preventive Medicine, 40, 293-298.

Tudor-Locke, C., Hatano, Y., Pangrazi, R. P., \& Kang, M. (2008). Revisiting "how many steps are enough?". Medicine and Science in Sports and Exercise, 40, 537-543.

Ulrich, B., \& Ulrich, D. (1985). The role of balancing in performance of fundamental motor skills in 3-, 4- and 5-years-old children. In C. JE \& H. J (Eds.), Motor developmental Current selected research. (pp. 87-97). Princeton: Princeton Book Co; 1985.

Wallen, E. F., Mullersdorf, M., Christensson, K., Malm, G., Ekblom, O., \& Marcus, C. (2009). High prevalence of cardio-metabolic risk factors among adolescents with intellectual disability. Acta Paediatrica, 98, 853-859.

Walsh, P. N., Kerr, M., \& van Schrojenstein Lantman-de Valk, H. M. (2003). Health indicators for people with intellectual disabilities: a European perspective. European Journal of Public Health, 13, 47-50. 
van Emmerik, R. E., Sprague, R. L., \& Newell, K. M. (1993). Quantification of postural sway patterns in tardive dyskinesia. Movement Disorders, 8, 305-314.

Wilmore, J., \& Costill, D. (1999). Physiology of sport and exercise: Champaign, IL: Human Kinetics .

Voelcker-Rehage, C., Godde, B., \& Staudinger, U. M. (2010). Physical and motor fitness are both related to cognition in old age. European Journal of Neuroscience, 31, 167-176.

World Health Organization. (2010). Global recommendations on physical activity for health. ISBN: 978-92-4159-997-9.

Åstrand, P. (2012). Ergometri Fitness test. Monark Exercise AB. Retrieved October 18, 2012 from http://www.monarkexercise. se/wk_custom/documents/\%7B3d8acc85c90a-4112-b677-b08f06e6cd49\%7D astrand_sv.pdf. 